\section{Kurt Harald Backus}

\author{
Joachim W. Deitmer, Kaiserslautern
}

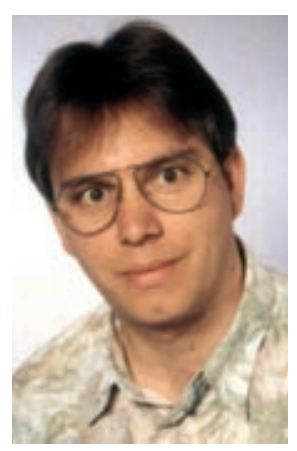

Kurt Harald Backus, Jahrgang 1958, Professor für Physiologie an der Johann Wolfgang Goethe-Universität Frankfurt, verstarb am 15. Juli 2005 nach langer, schwerer Krankheit.

Nach dem Biologie- und SportStudium in Heidelberg promovierte Harald Backus bei Helmut Kettenmann am Institut für Neurobiologie von Melitta Schachner über GABA- und Glutamatrezeptoren in
Astrozyten. Es schlossen sich PostdocAufenthalte in Basel bei Hoffmann-La Roche im Labor von Gerd Trube sowie im Pharmakologischen Institut der Universität Zürich bei Hanns Möhler an, bevor er als wissenschaftlicher Assistent an den Fachbereich Biologie der Universität Kaiserslautern kam. Dort vertiefte Harald Backus seine Forschung über die Charakterisierung von Transmitterrezeptoren in Gliazellen. 1996 habilitierte sich Harald Backus im Fachbereich Biologie der Universität Kaiserslautern für das Fach Zoologie. Nach einem weiteren Aufenthalt als Arbeitsgruppenleiter in der Abteilung von Heinz Wässle am Max-Planck-Insitut für Hirnforschung in Frankfurt nahm Harald
Backus 2001 einen Ruf auf eine C3-Professur für Physiologie in Frankfurt an.

Harald Backus war ein begeisterter Forscher und Lehrer, der es verstand, Studenten für die Neurobiologie und für seine Projekte zu gewinnen. Zuletzt beschäftigte er sich mit der wechselseitigen Modulation von Rezeptoren im Zentralnervensystem und deren Auswirkung auf die Aktivität von Neuronen, vor allem im visuellen und auditorischen System von Säugern. Harald Backus war ein sehr geschätzter Kollege, der an vielen wissenschaftlichen und kulturellen Dingen interessiert war, und der geistreich zu diskutieren verstand. Unvergessen auch seine Radtouren in der Pfalz und in Baden und die Besuche bei Radrennen, neben dem Schwimmen eine seiner Leidenschaften. Stets suchte er die Herausforderung, er war eine echte Kämpfernatur.

Harald Backus hinterlässt Frau und drei Kinder.

\title{
STELLENMARKT
}

\section{V) An der Universität Regensburg werden

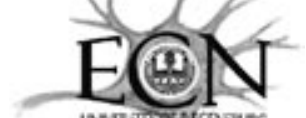

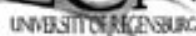 \\ 6 wissenschaftliche Mitarbeiter $(B A T) \mathrm{la}, \mathrm{m} / \mathrm{w})$ \\ in den Neurowissenschaften ab März 2006 für 5 Jahre gesucht. \\ Die Bewerber sollen sich durch exzellente wissenschaftliche Leistungen auf einem der genannten Gebiete und Freude an der Ausbildung im Rahmen des Elitenetzwerks Bayern geförderten Internationalen Elitestudienganges „Experimental and Clinical Neurosciences" auszeichnen: \\ BAT Ila Behavioural Neuroendocrinology (Gruppe Prof. Neumann) \\ BAT Ila Molecular Neuroendocrinology (Gruppe Prof. Neumann) \\ BAT Ila Molecular Neurogenetics (Gruppe Prof. Schneuwly) \\ BAT lla Neuroimaging (Gruppe Prof. Greenlee) \\ BAT Ila Cellular Neuroplasticity (Gruppe Prof. Winkler) \\ BAT Ila Behavioural Neuroplasticity (Gruppe Prof. Hajak)}

Voraussetzungen sind Promotion, Englischkenntnisse und nachweisbare Leistungen (Publikationen) auf dem Fachgebiet. Weibliche und behinderte Bewerber werden bei gleicher Eignung bevorzugt; Universitäts-eigene Krippe und Kindergarten sind vorhanden.

Schriftliche Bewerbungen sind mit CV, Lichtbild, Publikationsliste, Darstellung des wissenschaftlichen Werdeganges/ Schwerpunktes und Bezeichnung der Stelle bis zum 31. Januar 2006 zu senden an:

Prof. Dr. Inga Neumann (Koordinatorin des Elitestudienganges ECN) Institut für Zoologie, Universität Regensburg, 93040 Regensburg

weitere Informationen unter: $\quad$ www-elite-neurosciences.uni-regensburg.de inga.neumann@biologie.uni-regensburg.de 